\title{
RADIO POLARIZATION STUDIES OF SOME SOUTHERN GALAXIES
}

\author{
J.I. HARNETT ${ }^{1,3}$, R.F. HAYNES ${ }^{2}$, R. WIELEBINSKI ${ }^{3}$, U. KLEIN ${ }^{4}$ \\ 1. University of Sydney, 2. Australia Telescope National Facility \#, \\ 3. Max-Planck-Institut für Radioastronomie. 4. University of Bonn
}

\begin{abstract}
We have begun a long-range project to study southern galaxies using the radio telescopes at Parkes and Molonglo, the Siding Spring optical facilities and soon, the Australia Telescope. Here we present the results of polarization mapping at two wavelengths of the galaxies NGC 55, 253, 4945, M 83 and the Circinus Galaxy.
\end{abstract}

\section{OBSERVATIONS AND RESULTS}

We have observed the galaxies at wavelengths of $6.3 \mathrm{~cm}$ and $3.5 \mathrm{~cm}$ ( 4.75 and $8.55 \mathrm{GHz}$ ) with the Parkes $64 \mathrm{~m}$ radio telescope using a prototype Australia Telescope receiver and a modified MPIfR Polarimeter (Haynes et al.,1989). Beamsizes were 4.5' $(4.75 \mathrm{GHz})$ and $2.7^{\prime}(8.55 \mathrm{GHz})$. The observations were made in January 1989 under variable weather conditions and data reduction procedures followed those described by Harnett et al.(1989) for NGC 4945. Each of the maps is discussed below.

NGC 55 Continuum emission at $6.3 \mathrm{~cm}$ extends over the whole of the optical image with a peak of $157 \mathrm{mJy}$. A weak extension is seen to the south. Polarized radiation is marginally detected.

NGC 253 Radio continuum emission aligned with the optical disk of this edge-on ( $i=75^{\circ}$ ) galaxy is seen at both wavelengths. Polarized radiation is seen as two maxima on opposite sides of the nucleus with Evectors perpendicular to the disk. This agrees with the published polarization map of NGC 253 at $2.8 \mathrm{~cm}$ (Klein et al., 1983).

NGC 4945 The $6.3 \mathrm{~cm}$ map showed two polarization maxima on opposite sides of the nucleus (Harnett et al., 1989). The $3.5 \mathrm{~cm}$ map confirms this result and shows that there is minimal Faraday rotation in the galaxy at shorter wavelengths. Two extensions away from the nucleus seen at $6.3 \mathrm{~cm}$ are confirmed at $3.5 \mathrm{~cm}$. This is significant as it is indicative of polarized emission away from the galactic plane $\left(i=80^{\circ}\right)$. Figure 1 shows the 6.3 and $3.5 \mathrm{~cm}$ total power maps with E-vectors whose lengths are proportional to the polarized intensity.

\footnotetext{
\# The Australia Telescope National Facility is operated in association with the Division of Radiophysics by CSIRO.
} 
M 83 The $6.3 \mathrm{~cm}$ result for the face-on galaxy M 83 agrees well with the observations of Sukumar et al.(1987), even though our resolution is poorer. E-vectors, turned by $90^{\circ}$ to approximate the magnetic field direction, closely follow the spiral arm pattern. Maximum polarization is located to the south-east and north-west of the optical bar, while the minima are in the vicinity of the ends of the bar, a region where one would expect random field orientations to be more likely. There is only marginal evidence for polarization at $3.5 \mathrm{~cm}$.

CIRCINUS This "hidden" galaxy is seen as a slightly extended source and is linearly polarized at both 6.3 and $3.5 \mathrm{~cm}$. The position angle (PA) of the E-vectors is very different $\left(\mathrm{PA} \sim 55^{\circ}\right.$ at $6.3 \mathrm{~cm}, \mathrm{PA} 132^{\circ}$ at $3.5 \mathrm{~cm})$; probably a result of high Faraday rotation in the plane of the galaxy.

\section{DISCUSSION}

These new polarization maps of southern galaxies show the presence of magnetic fields in at least four objects. At our angular resolution, one of the main features noted for a number of the galaxies are the two polarization maxima on opposite sides of the nucleus. This is certainly the case for the nearly edge-on galaxies NGC 253 and NGC 4945. It could be a geometrical effect, where the line-of-sight is perpendicular to the magnetic field in the opposite spiral arms. In these directions the Faraday rotation is minimal and hence the polarized intensity greatest. However, in view of results for numerous face-on galaxies (e.g. M 83), in which two maxima are also found on opposite sides of the nucleus (see experimental papers in this volume) a physical explanation is also possible. Higher angular resolution observations (with the compact array of the Australia Telescope) are necessary to follow up these studies.

\section{REFERENCES}

Harnett J.I., Haynes R.F., Klein U., Wielebinski R., 1989. Astron. Astrophys. (in press)

Haynes R.F., Murray J.D., Hunt A.J., Wark R., Sciacca L, Wayte S., Klein U., Wielebinski R., Buczilowski U.R. 1989, Proc. Astron. Soc. Aust. (in press)

Klein U., Urbanik M., Beck R., Wielebinski R., 1983. Astron. Astrophys.s 127, 177

Sukumar S., Klein U., Grăve R., 1987: Astron. Astrophys., 184, 71
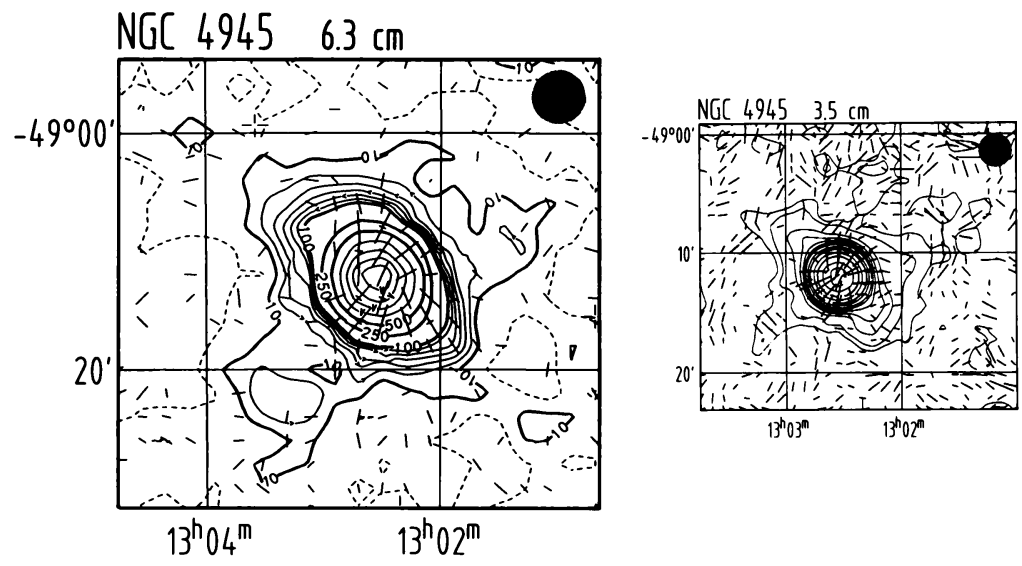

Figure 1. The total power maps of NGC 4945 with E-vectors superimposed. 